\title{
AVALIAÇÃo ASSISTIDA PARA CRIANÇAS COM NECESSIDADES EDUCACIONAIS ESPECIAIS: UM RECURSO AUXILIAR NA INCLUSÃO ESCOLAR ${ }^{1}$
}

\author{
DYNAMIC ASSESSMENT FOR CHILDREN WITH SPECIAL EDUCATIONAL NEEDS: AN \\ AUXILIARY RESOURCE IN THE SCHOOL INCLUSION
}

Sônia Regina Fiorim ENUMO²

\begin{abstract}
RESUMO: a inclusão escolar dos portadores de necessidades educativas é proposta dominante na Educação Especial e na Educação em geral nas últimas décadas, direcionando programas e políticas educacionais e de reabilitação em vários países, incluindo-se o Brasil. Exige a transformação da escola, defendendo a inserção de alunos com quaisquer necessidades no ensino regular, cabendo às escolas se adaptarem às características dos alunos, o que leva à ruptura com o modelo tradicional de ensino. Reside aqui o mérito e o início dos problemas. As instituições de Ensino Fundamental têm encontrado dificuldades na inclusão escolar, a começar pelo diagnóstico das dificuldades de aprendizagem, terminando na pouca capacitação profissional para atender pedagógica e psicologicamente essa população. Procurando contribuir neste aspecto básico do processo inclusivo - o diagnóstico da criança com necessidades educativas especiais -, a avaliação assistida se apresenta como uma modalidade de avaliação complementar à avaliação tradicional de habilidades cognitivas e lingüísticas. Esta inclui ajuda do examinador durante o processo, após uma fase inicial sem ajuda, sendo comum em pesquisa o procedimento teste-ajuda-reteste, em que o sujeito funciona como seu próprio controle. Esta modalidade de avaliação do processamento cognitivo e lingüístico favorece o desempenho e se apresenta como mais prescritiva à adaptação curricular. Utilizada especialmente na Educação Especial, esta é uma proposta de avaliação ainda pouco utilizada no Brasil, onde se encontram tipicamente trabalhos de pesquisa desde a década de 90 do século passado.
\end{abstract}

PALAVRAS-CHAVE: avaliação assistida; inclusão escolar; crianças com necessidades educacionais especiais.

\begin{abstract}
Special Education and Education in general, in the last decades, addressing programs and educational and rehabilitation politics in several countries, including Brazil. It demands the transformation of school, proposing the students' insertion with any needs in the regular school, letting to the schools the adaptation to the students' characteristics, what makes the rupture to the traditional model of teaching and is the problem starting. The institutions of Basic School have been having difficulties in the school inclusion, starting in the diagnosis of the learning disabilities, finishing in the little professional training to assist pedagogically and psychologically that population. Trying to contribute in this basic aspect of the inclusive process - the child's diagnosis with special educational needs -, the dynamic assessment is a complementary modality to the traditional evaluation of cognitive and linguistic abilities. This includes the examiner's help during the process, after an initial phase without help, being common in research works the procedure test-aided-retest, in that the subject works as his own control. This evaluation modality of the cognitive and linguistic processing favors the acting and it comes as more prescriptive to the curriculum adaptation. Especially used in the Special Education, this is still a little used evaluation proposal in Brazil, where there are research papers since the 90s last century.
\end{abstract}

KEYWORDS: dynamic assessment; school inclusion; children with special educational needs.

\footnotetext{
${ }^{1}$ Financiamento: CNPq (Proc. $n^{\circ}$ 521014/2003-5), FACITEC - Prefeitura Municipal de Vitória.

${ }^{2}$ Universidade Federal do Espírito Santo (UFES), Departamento de Psicologia Social e do Desenvolvimento, Programa de Pós-Graduação em Psicologia - soniaenumo@terra.com.br
} 


\section{INTRODUÇÃO}

A inclusão dos portadores de necessidades educativas especiais (NEE) tem sido proposta norteadora e dominante na Educação Especial e da Educação em geral, direcionando programas e políticas educacionais e de reabilitação em vários países, incluindo-se o Brasil. É sucessora da integração, a qual era vista como um conceito novo para uma prática antiga. Historicamente, a proposta de integração escolar foi elaborada em 1972, na Educação Especial, por um grupo de profissionais da Escandinávia, liderados por Wolfensberger, na forma do chamado 'princípio de normalização'. Este princípio apregoa que todas as pessoas portadoras de deficiências têm o direito de usufruir condições de vida o mais comum ou "normal" possível, na sociedade em que vivem. Dito de outra forma, normalizar não quer dizer tornar normal, mas significa dar à pessoa oportunidades, garantindo seu direito de ser diferente e de ter suas necessidades reconhecidas e atendidas pela sociedade (CARDOSO, 1992; CARVALHO, 1994; GLAT, 1998). Assim, o conceito de 'integração' é uma das conseqüências fundamentais do 'princípio de normalização', proposto como objetivo, enquanto a integração seria o processo, que deveria ir muito além de colocar ou manter excepcionais em classes regulares. E, portanto, parte fundamental de todo o processo educacional.

As noções de normalização e integração se fortaleceram no final dos anos 60 e início dos anos 70 do século passado, junto com os movimentos de direitos civis de minorias e grupos marginalizados, difundindo-se pelos Estados Unidos da América, Canadá e diversos países da Europa, influenciando os teóricos e profissionais no Brasil (GLAT, 1998; NUNES et al., 1998; SAINT-LAURENT, 1997).

Herdando essa política de integração, tendo como ponto de partida os resultados positivos alcançados com a prática da inclusão escolar nos países desenvolvidos nas duas últimas décadas, o sistema educacional brasileiro tem vivenciado um momento de transição no atendimento do aluno com NEE. O modelo de Educação Especial anterior adotava as 'classes especiais' para alunos com NEE, propondo-se a um atendimento mais específico, mas, acabou por contribuir para a segregação dessas crianças. Dessa forma, a inclusão escolar ganhou força, refletindo os esforços das sociedades atuais pela sua integração em salas regulares de ensino, de forma a aceitar e respeitar suas diferenças. Assim, a partir do final dos anos 80 do século passado, o termo integração começou a perder força, sendo substituído pela idéia de inclusão, uma vez que o objetivo é incluir, sem distinção, todas as crianças, independentemente de suas habilidades. A inclusão exige a transformação da escola, pois defende a inserção no ensino regular de alunos com quaisquer déficits e necessidades, cabendo às escolas se adaptarem às necessidades deles, ou seja, a inclusão acaba por exigir uma ruptura com o modelo tradicional de ensino (KARAGIANNIS; STAINBACK; STAINBACK, 1999a; MARCHESI; ECHEITA; MARTÍN, 1995; SASSAKI, 1997).

Reside aqui o mérito dessa proposta e o início dos seus problemas. Entre vários obstáculos à inclusão de alunos com NEE, na prática, percebe-se que, 
mesmo aquelas crianças que se encontram inseridas no sistema regular de ensino, continuam sendo isolados dos seus companheiros de turma não-deficientes. Pesquisas têm demonstrado que esses alunos encontram-se incluídos fisicamente, mas não social e emocionalmente, (BATISTA; ENUMO, 2004; MARCHESI; ECHEITA; MARTÍN; 1995; MENDES; FERREIRA; NUNES, 2003), dados esses consistentes com aqueles identificados na literatura desde as primeiras tentativas de integração na década de 50 do século XX (AUSUBEL; HANESIAN; NOVAK, 1978). De outro lado, o processo de inclusão de crianças com deficiência no ensino regular possibilita-lhes estar interagindo espontaneamente com situações diferenciadas, enquanto adquirem conhecimento e se desenvolvem, com efeitos na aquisição de habilidades sociais, da vida diária e de comunicação (KARAGIANNIS; STAINBACK; STAINBACK, 1999b). Essa inclusão, entretanto, não deve ser facilmente resolvida a partir de uma resolução de cunho legal ou teórica, uma vez que variáveis relacionadas a processos grupais e reações de preconceito podem influenciá-la, seja facilitando ou dificultando a interação dessas pessoas com aquelas ditas "normais" (STAINBACK; STAINBACK, 1999).

É necessário que, na prática, seja adotada uma política educacional que promova mudanças curriculares, efetivando a participação dos pais no processo de inclusão e capacitando as escolas e os profissionais que lidam com essas crianças. Nesse sentido, é de fundamental importância que ocorra integração ou parceria entre a universidade e as instituições públicas de Ensino Fundamental. Estas últimas têm encontrado dificuldades na inclusão escolar dessas crianças, a começar do processo de diagnóstico das dificuldades de aprendizagem, terminando na pouca capacitação profissional para atender pedagógica e psicologicamente essa população (MARCHESI; ECHEITA; MARTÍN, 1995; MENDES; FERREIRA; NUNES, 2003).

Procurando contribuir em um desses aspectos básicos do processo de inclusão - o diagnóstico das dificuldades de aprendizagem desses alunos com NEE - discute-se, a seguir, as possibilidades de uma modalidade de avaliação psicológica que tem se mostrado bastante útil à Educação Especial - a avaliação assistida (STERNBERG; GRIGORENKO, 2002).

\section{AvaliaÇão PSICOLÓGICA DE CRIANÇAS COM NECESSIDADES EDUCATIVAS ESPECIAIS}

Tomando como referência o fato de que a criança com NEE pode representar grande ônus sócio-afetivo familiar e pessoal e, também, econômico para a sociedade, é importante que sejam adotadas medidas que minimizem essa situação. Tais medidas podem incluir, entre outras, a adoção de procedimentos de avaliação mais adequados às necessidades especiais da criança deficiente, como a avaliação "assistida" ou "dinâmica" (CAMPIONE, 1989; HAYWOOD, TZURIEL, 2002; LINHARES, 1995; TZURIEL, 2001), além da capacitação de pais e profissionais para implementar estratégias que promovam o desenvolvimento infantil. 
O processo de identificação e avaliação de deficiências é uma etapa primordial (MARCHESI; MARTÍN, 1995). Esse papel, entretanto, em certas circunstâncias, pode ser perigoso. Diagnosticar significa "procurar através da" análise de sintomas e sinais, utilizando recursos técnicos, a fim de localizar as causas e prescrever tratamentos para os respectivos quadros identificados. O diagnóstico é considerado a base para o encaminhamento a esse tipo de atendimento, sendo feito, segundo portaria ministerial, por órgão público competente, que procede a avaliação das condições físicas, mentais, psicossociais e educacionais do indivíduo, visando a estabelecer o prognóstico e a programação terapêutica e educacional (ENUMO; CUNHA, 2001). Contudo, segundo os estudiosos da construção social da deficiência, observa-se que o diagnóstico pode levar a estruturação de estigmas, que funcionarão como um rótulo imputado aos indivíduos identificados como desviante ou anormal, que passarão a integrar o grupo minoritário e, conseqüentemente, marginalizado dos deficientes (AMARAL, 1995; GOFFMAN, 1982). Logo, o diagnóstico deve ser um processo contínuo e sistemático de avaliação, cujo objetivo é investigar interpretar, orientar e, sobretudo, prevenir a estigmatização de indivíduos identificados por suas diferenças.

Analisando os usos da avaliação psicológica, Mofese e Acheson (1997) consideram que, especialmente no campo do desenvolvimento humano, ela é extremamente útil quando se deseja: a) conhecer o desempenho e as potencialidades do indivíduo; b) avaliar os progressos ou resultados de programas de intervenção; c) identificar a fonte de problemas escolares; d) realizar investigações na área de diagnóstico e prevenção de distúrbios/atrasos no desenvolvimento. Esses autores inclusive defendem o uso de testes mentais padronizados como parte ou base para "baterias de triagem" usadas para a identificação precoce de distúrbios no desenvolvimento ou de crianças de risco.

A avaliação das aptidões cognitivas constitui-se, assim, uma das áreas mais significativas da aplicação da Psicologia, segundo Almeida (1996). Este fato pode ser confirmado por levantamentos internacionais, feitos no início da década de 90 do século passado, mostrando que os testes relacionados à avaliação da inteligência de crianças e jovens estavam entre os dez testes mais citados, com o WISC-III, em primeiro lugar geral (ALMEIDA, 1996; CARNEIRO; FERREIRA, 1992; HUTZ; BANDEIRA, 1993). Contudo, lembram os autores nacionais que, apesar desse uso freqüente, há uma pequena e assistemática publicação na área, no Brasil.

De toda maneira, esse uso geral de instrumentos psicométricos tem sobrevivido, mas acompanhado de grande polêmica, seja pela indeterminação do conceito de inteligência, gerada tanto por questões teóricas, por ser um conceito altamente influenciado pelo contexto social do momento, seja pela pluralidade de métodos e instrumentos utilizados na sua medição (COLL; ONRUBIA, 1996).

A crítica básica ao uso de testes padronizados de inteligência tem sido centrada no fato de que eles podem ser culturalmente enviesados contra os pobres e os grupos minoritários, devido à diferenças na cultura, na linguagem, nos valores 
e no background de experiências, além da inadequação técnica e científica desses testes de inteligência - viés do teste e do procedimento de validação usado na construção e padronização - e da questão ética relacionada às conseqüências educacionais e sociais do uso desses testes. Os testes de inteligência teriam como fontes de erro, segundo síntese de Reynolds (apud UTLEY, HAYWOOD; MASTERS, 1992): a) conteúdo inapropriado, derivado do ambiente, vocabulário e valores da classe média branca; b) amostras de padronização inapropriadas, com sub-representação de minorias étnicas; c) vieses de linguagem e do examinador, que, em geral são brancos e consideram apenas a língua dominante; d) falta de equiparação de conseqüências sociais - os resultados e rótulos derivados dos testes acentuam de forma desproporcional as desvantagens educacionais e do mercado vocacional dessa minoria; e) medida de constructos diferentes - relacionado ao item a, considera-se que os teste medem diferentes atributos quando usados com crianças diferentes da amostra original, não sendo válidos, portanto, para avaliar a inteligência dos grupos minoritários; g) validade preditiva diferencial, sendo útil somente para a classe média branca, sem predizer de modo confiável qualquer comportamento relevante das minorias, assim como ocorre com os testes acadêmicos. Cria-se, assim, a questão de interesse, principalmente para educadores, cientistas sociais e geneticistas do comportamento, de saber se as diferenças no QI (variável manifesta - o escore no teste de inteligência) refletem diferenças na inteligência em si (variável latente, não observável, que é assumida pela medida do teste) (UTLEY, HAYWOOD; MASTERS, 1992).

Com base nessas críticas, muitos psicólogos que atuam em diversas áreas de atendimento a pessoas com deficiência - escolar, clínica, desenvolvimento, social, orientação vocacional, organizacional - preferem até se abster de realizar esse tipo de atendimento, sentindo-se desconfortáveis com a situação, questionando os instrumentos, sua validade, fidedignidade, eficácia, inclusive não sabendo como se comportar com essas pessoas (AMIRALIAN, 1997). Em situação pior, alguns psicólogos educacionais entregam laudos psicológicos que nem apresentam a criança avaliada, mas incluem apenas os resultados de QI, afirmando a deficiência da criança, "culpando a vítima" e legitimando cientificamente o preconceito, com completo desconhecimento do que se passa na escola, como relatam Machado, Souza e Sayão (1997).

Afora a má-formação profissional, as evidências empíricas não apóiam a alegação de que as diferenças nos resultados dos testes das crianças de grupos minoritários sejam devidas aos aspectos de sua validade psicométrica, seja de conteúdo, preditiva ou de critério e de constructo. A qualidade dos instrumentos mais utilizados, como o WISC-III e as Matrizes Progressivas de Raven, por exemplo, não têm vieses de teste relacionados à validade de conteúdo, preditiva ou de constructo (UTLEY; HAYWOOD; MASTERS, 1992).

Assim, segundo esses autores, a explicação para as diferenças individuais na inteligência deve estar em outra variável além das qualidades psicométricas dos testes, pois as diferenças entre os grupos sociais persistem seja em testes verbais ou não-verbais, com (culture-fair) e sem conteúdo cultural (culture- 
free) (condição praticamente impossível de separar), de inteligência ou de aquisição acadêmica. Por exemplo, os fatores não-intelectuais, como a motivação intrínseca, a necessidade de domínio, o locus de controle, a ansiedade, a tolerância à frustração, a autoconfiança e a acessibilidade à mediação podem influenciar o desempenho cognitivo e não são considerados nos testes psicométricos (MARTÍN, MARCHESI, 1995; TZURIEL, 1991, 2001). Assim, “[...] o problema não está no que os testes padronizados fazem e eles o fazem bem, mas no que eles não fazem" (TZURIEL, 2001, p. 4).

Bem entendida a questão da qualidade dos instrumentos, Lidz (1991) entende que a própria avaliação é um processo cognitivo em si e que os especialistas em diagnóstico devem responder à pergunta: "O que nós precisamos saber e o que irá produzir a informação apropriada?" (p. xi). Se a questão é saber como os estudantes estão em relação ao desempenho de seus pares em idade ou em grau de escolaridade, o procedimento a ser escolhido deve ser referenciado à norma. Se a questão diagnóstica refere-se à localização das habilidades ou do nível de desenvolvimento do estudante dentro de uma pré-determinada hierarquia de comportamentos, devem ser escolhidos procedimentos referenciados ao desenvolvimento. Quando a pergunta relaciona-se ao conhecimento específico básico que o aluno adquiriu ou não, o procedimento escolhido deve ser referenciado $a$ critério ou ao currículo. E se a pergunta que orienta o processo diagnóstico refere-se à responsividade do aprendiz à intervenção, o uso de processo de solução de problemas, o procedimento a ser escolhido é a avaliação dinâmica ou assistida. Adotando-se um modelo ecologicamente válido, essa autora considera que procedimentos adicionais devem ser incluídos para avaliar as contribuições ambientais ao desempenho observado. Nesse sentido, “[...] nenhum desses procedimentos avaliativos é inerentemente melhor do que outro" (p. xi); nem deve ser usada uma única abordagem para todas as perguntas (LIDZ, 1991).

Segundo Pearce (1995), crianças com NEE estão em situação de risco de desenvolvimento ou de problemas psicológicos, geralmente por sua dificuldade em aprender sozinho e limitação na capacidade de generalização ou de transferência de aprendizagem. Tais problemas, por sua vez, decorrem de dificuldades para planejar e regular seus processos de aquisição de conhecimento (MARTÍN; MARCHESI, 1995). Parece extremamente relevante, por conseguinte, conhecer seu potencial de aprendizagem, sua capacidade de tirar proveito da instrução oferecida, saber o tipo de ajuda que lhe é útil, indicando, assim, o papel do professor. Em última instância, é a "avaliação assistida", "dinâmica" ou "interativa", considerada adequada e útil para essa população, mostrando-se como uma abordagem interativa, otimista, precisa e prescritiva, com benefícios de longo prazo (TZURIEL; HAYWOOD, 1992). 


\section{AvaliaÇão assistida - uma proposta complementar na aValiaÇão de CRianças COM} NECESSIDADES EDUCATIVAS ESPECIAIS

Haywood e Tzuriel (2002) apresentam a avaliação assistida como uma inovadora abordagem para avaliar habilidades humanas, em particular o potencial de aprendizagem, visando basicamente à identificação e remoção de barreiras nãointelectuais à expressão da inteligência.

A avaliação assistida pode ser concebida como um conceito amplo, que vai além de técnicas e procedimentos específicos, pois inclui várias teorias, metodologias, funções e objetivos, relacionando-se a novas concepções de inteligência e a várias propostas de avaliação: a "propensibilidade" à aprendizagem, de Feuerstein (1979) e Tzuriel (2001); os procedimentos de "dicas graduadas" (graduated prompts assessment), de Campione e Brown (1987); o treino de procedimentos para testes de avaliação do potencial de aprendizagem (learning potential assessment), de Budoff (1987); a avaliação interativa, de Lidz $(1987,1991)$ e de Haywood (HAYWOOD; TZURIEL, 1992); a abordagem ao "teste de aprendizagem" (learning test) de Guthke e Wingenfeld (1992); a reestruturação dos dados em presença do teste, de Carlson e Wiedl (1992); a indução de estruturas lógicas (Paour, 1992); a "perspectiva transacional da avaliação psicoeducacional" proposta por Tzuriel (HAYWOOD; TZURIEL; VAUGH, 1992); a "avaliação psicopedagógica dinâmica" de Fonseca (2001); e a avaliação do processamento cognitivo, de Swanson (Swanson Cognitive Processing Test- S-CPT) (STERNBERG; GRIGORENKO, 2002).

Sua origem está datada na década de 70 do século passado, com os trabalhos de Reuven Feuerstein e seus colegas, em Israel, insatisfeitos com os métodos de avaliação tradicionais em fornecerem informações sobre a habilidade para aprender dos estudantes. Passaram, então, a desenvolver esta modalidade de avaliação voltada ao fornecimento de informações que possam ser diretamente aplicadas na prática por educadores e psicólogos clínicos e escolares (CAMPIONE, 1989; HAYWOOD; TZURIEL, 1992, 2002; LIDZ, 1989, 1991; LINHARES, 1995; TZURIEL, 2001; STERNBERG; GRIGORENKO, 2002). Partia-se da premissa de que deveriam ser consideradas todas as explicações alternativas para um pobre desempenho em aprendizagem e solução de problemas antes de inferir que são decorrentes de uma incapacidade para aprender (UTLEY; HAYWOOD; MASTERS, 1992). Considerava-se também que "[...] QI não é sinônimo de habilidade para aprender" (LIDZ, 1991, p. 4). Assim, segundo Haywood, Tzuriel e Vaught (1992), o conceito de inteligência, entendida como uma "habilidade inata", não era suficiente para explicar as diferenças individuais no pensamento, percepção, aprendizagem, solução de problemas e interação social. Era necessário, então, adotar concepções teóricas sobre a plasticidade da cognição humana para atender à necessidade prática de encontrar novas medidas de diagnóstico para crianças que não se saíam bem em testes convencionais. 
De acordo com a revisão do tema feita por Lidz (1987) e Tzuriel (2001), foi a introdução da teoria de Vygotsky por Brown e Ferrara e das idéias de Feuerstein por H.C. Haywood, na Psicologia norte-americana, que impulsionaram as pesquisas sobre avaliação assistida, seguindo a proposta original de Alfred Binet, o qual defendia a avaliação de 'processos' mais do que 'produtos' de aprendizagem. Assim, os conceitos de 'zona de desenvolvimento proximal' (ZDP), de Vygotsky, e a 'teoria de aprendizagem mediada' (MLE), de Feuerstein, segundo Tzuriel (2001), formam a base conceitual para a elaboração da avaliação assistida, que procura incluir fatores sócio-culturais na compreensão do desenvolvimento cognitivo e do potencial de aprendizagem.

Tzuriel (2001, p. 6), um dos principais representantes dessa abordagem, define a avaliação assistida como "[...] uma avaliação do pensamento, percepção, aprendizagem e solução de problemas por meio de um ativo processo de ensino voltado para modificar o funcionamento cognitivo". Difere dos testes convencionais por seus objetivos, processos, instrumentos, situação de teste e interpretação dos resultados (CAMPIONE, 1989; FONSECA, 2001; LINHARES, 1995).

A abordagem "clínica" da avaliação assistida, proposta por Feuerstein e Tzuriel, em Israel, foi pioneira, sendo seguida pelos psicólogos do Reino Unido (ELLIOTT; LAUCHLAN; STRINGER, 1996). Nos EUA e na Europa e, especialmente no contexto da pesquisa, inclusive aqui no Brasil, tem sido utilizado um modelo de aplicação mais padronizado, "estruturado". Este é traduzido na seqüência "testeintervenção-reteste", quando usado na aplicação, podendo haver também uma estruturação na pontuação dos resultados. Assim, é possível não só observar os ganhos da criança após a ajuda, mas, também, verificar se a aprendizagem se mantém, quando a tarefa é reapresentada sem ajuda, servindo a criança como seu próprio controle (CAMPIONE, 1989; ELLIOTT, LAUCHLAN; STRINGER, 1996; ENUMO; LINHARES; ESCOLANO, 2004; LIDZ, 1991; LINHARES, 1995; LINHARES; ESCOLANO; ENUMO, no prelo).

Independentemente do modelo, a interação visa a otimizar o funcionamento típico do examinando e não apenas obter uma amostra deste ou avaliar a inteligência. Assim, pretende-se compreender como a criança aprende, facilitando de modo direto a competência cognitiva pela criação de uma situação em que a criança se envolve processo de aprendizagem (LIDZ, 1996). Dessa maneira, segundo Lidz (1991) e Tzuriel (2001), é possível obter informações sobre processos metacognitivos funcionais, disfuncionais e em vias de desenvolvimento (no caso de pré-escolares), assim como sobre a quantidade e tipo de intervenção necessário para produzir mudanças. Produz-se, portanto, sugestões para intervenção que podem facilitar e melhorar o desempenho da criança.

As áreas da Educação, Educação Especial, Psicopatologia, Neuropsicologia, assim como o estudo de diferenças culturais e desenvolvimento humano, têm sido beneficiadas com o uso da avaliação assistida como ferramenta clínica e de pesquisa. No geral, sua aplicação é individualizada; mas, há aplicações 
em grupo (RAND; KANIEL, 1987; TZURIEL; FEUERSTEIN, 1992) e computadorizada (TZURIEL; SHAMIR, 2002). Há relatos de uso em diversos contextos de avaliação: crianças em risco de fracasso escolar, em desvantagem social, com retardo mental, surdez, altas habilidades, problemas emocionais, dificuldades de aprendizagem (crianças e adultos), pré-escolares, estudantes universitários, penitenciários, pacientes com danos cerebrais, esquizofrênicos, adolescentes psicóticos, minorias culturais, na análise da mediação pais-criança, na verificação de habilidades lingüísticas de narrativa; na avaliação de programas de educação cognitiva, a exemplo do Bright Start de H.C. Haywood, e de educação pré-escolar (HAYWOOD; MILLER, 2003; HAYWOOD; TZURIEL, 1992, 2002; LIDZ, 1989, 1991; MILLER, GILLAM; PEÑA, 2001; TZURIEL, 2001).

No Brasil, pesquisas sobre avaliação assistida têm sido desenvolvidas desde o início da década de 90 do século passado, segundo revisão de Linhares, Escolano e Enumo (no prelo) e Linhares e Enumo (no prelo).

As pesquisas sobre avaliação cognitiva assistida têm sido realizados no país com diferentes tipos de sujeitos:

a) crianças com dificuldade de aprendizagem, por Santa Maria e Linhares (1999), Linhares (1996, 1998), Linhares, Santa Maria, Escolano e Gera (1998), Ferriolli (2000), Ferriolli et al. (2001), Gera (2001), na Universidade de São Paulo (USP) Ribeirão Preto, e por Dias (2004), Dias e Enumo (2004), na Universidade Federal do Espírito Santo (UFES) em atuação conjunta com a Universidade do Estado de Mato Grosso (UNEMAT);

b) alunos de primeira série do Ensino Fundamental, por Escolano $(2000,2004)$ e Escolano e Linhares (2000), na USP-Ribeirão Preto;

c) crianças com deficiência mental, por Santa Maria e Linhares (1999), na USPRibeirão Preto;

d) crianças prematuras, por Bordin (2005), Bordin, Linhares e Jorge (2001) e Linhares, Bordin e Carvalho (2004), na USP-Ribeirão Preto;

e) crianças com deficiência visual, por Batista, Horino e Nunes (2004), Cunha (2004), Cunha e Enumo (2003); Enumo e Batista (2000); Enumo, Batista e Ferrão (2005); Enumo et al. (2000); Fonseca e Cunha (2003); Fonseca, Cunha e Enumo (2002), em trabalhos conjuntos do Centro de Estudos e Pesquisas em Reabilitação (CEPRE) da Universidade Estadual de Campinas (UNICAMP) e UFES, além de intercâmbio internacional com o Professor Vitor da Fonseca, vinculado à Faculdade de Motricidade Humana (FMH), da Universidade Técnica de Lisboa; e

f) crianças com problemas de comunicação, por Paula (2004) e Paula, Enumo e Maia (2004), na UFES. 
Além da avaliação de habilidades cognitivas, a avaliação assistida também tem sido utilizada no país em outros contextos:

a) para a avaliação de habilidades lingüísticas da narrativa em crianças com câncer, por Motta et al. (2005) e em pré-escolares, por Leite et al. (2004), na UFES;

b) para a avaliação de efeitos de programas de intervenção - em criatividade, por Dias (2004) e Dias et al. (2003), programas com sistemas de comunicação alternativa, por Paula (2004) e Paula et al. (2003), na UFES, e para avaliação de programa de ensino regular, por Escolano (2000, 2004), na USP-Ribeirão Preto; e

c) para a avaliação de fatores não-intelectuais em provas assistidas para crianças com necessidades educativas especiais, por Ferrão et al. (2005) e Orlandi et al. (2004), na UFES.

Também foi usada a avaliação assistida para analisar a mediação materna em dois contextos: 1) durante a interação mãe-criança com deficiência visual em situação de jogo, por Cunha (2004), Cunha, Enumo e Pedroza (2004) e Fonseca e Cunha (2003), na UFES, em intercâmbio com a FMH; 2) durante a interação da mãe com a criança normal, por Linhares, Martins e Klein (2004) e Klein (2005), na USP-Ribeirão Preto.

Por fim, a avaliação assistida tem sido utilizada no país para analisar a mediação do psicólogo educacional durante a aplicação de programas de intervenção em crianças com necessidades especiais, por pesquisadores da UFES em intercâmbio com a UNEMAT na área da criatividade (DIAS; PAULA; FERRÃO; ENUMO, no prelo; PEREIRA; DIAS; ENUMO, 2003), e com a Universidade Federal do Rio de Janeiro, na área da deficiência visual (CUNHA, 2004; ENUMO et al., 2002).

Haywood e Tzuriel (2002) e Tzuriel (2001) encontraram conclusões confiáveis derivadas da avaliação assistida, a partir de estudos empíricos da área o processo de mediação ou ensino de estratégias lógicas, entre o pré e o pós-teste, leva a uma melhora no desempenho, diferentemente do mero ensino de conteúdo, do fornecimento de pistas para respostas corretas (graduated prompts) ou da nãointervenção. Todavia, depende do tipo de ensino, sua intensidade, natureza das barreiras cognitivas da pessoa e da distância psicológica entre o conteúdo de ensino e o conteúdo de desempenho dos testes, sendo os efeitos do ensino mais visíveis e efetivos em tarefas difíceis, complexas e/ou abstratas. O resultado principal de qualquer aprendizagem ou sua própria medida - a transferência de estratégias mediadas para a solução de novos problemas, ocorre mais facilmente para a transferência próxima do que para a distante, principalmente quando o ensino contém estratégias pedagógicas planejadas para a promoção da transferência, mostrando que esta não acontece ao acaso (HAYWOOD; TZURIEL, 2002).

Segundo Tzuriel (2001), a avaliação assistida prediz melhor o desempenho acadêmico para pessoas com retardo mental do que para aqueles sem o problema; além de oferecer uma melhor estimativa do potencial de 
aprendizagem do que quando comparada às estimativas feitas por testes estáticos, normativos.

A observação da aplicação de provas assistidas fornece mais informações úteis para professores do que a observação de provas estáticas ou normativas (BENJAMIN; LOMOFSKY, 2002; DELCLOS; BURNS; VYE, 1993; DELCLOS; BURNS; KULEWICZ, 1987). Pais de crianças com deficiência visual, ao observarem os dois tipos de avaliação, têm mostrado sua aprovação e melhor aceitação da avaliação assistida quando comparada com avaliações estáticas ou padronizadas (CUNHA, 2004; CUNHA et al., 2004).

Além disso, a avaliação assistida tem se mostrado muito útil como ferramenta de pesquisa nos casos de examinandos que relutam em responder, aqueles com sérios impedimentos de linguagem, os que bloqueiam, de modo psicopatológico, suas habilidades para se engajar efetivamente em tarefas de solução de problemas, casos de transculturalismo, de discriminação social, com conhecimento básico inadequado para a idade, autoconceito negativo como aprendiz, e hábitos improdutivos frente a situações de solução de problemas. Permite, assim, obter um conhecimento que é difícil de se obter com a utilização de testes normativos. Dessa forma, altera previsões pessimistas derivadas dos testes estáticos, principalmente quando combinada com programas de educação cognitiva ou qualquer outro voltado para melhorias na educabilidade de crianças e adolescentes. Também, permite fornecer dados sobre a "modificabilidade" cognitiva (cognitive modifiability) a partir da interação mãe-criança em pesquisas desenvolvimentistas e para a avaliação de programas de educação cognitiva; sendo útil para a habilitação ou reabilitação, inclusive para casos de danos cerebrais e desordens psiquiátricas. Sua efetividade, entretanto, depende de uma mediação da aprendizagem que especifique claramente as tarefas, vise às operações metacognitivas, recuse a ignorância como um indicador de inabilidade, dê atenção especial às barreiras afetivas/motivacionais e tenha expectativa de sucesso (HAYWOOD; TZURIEL, 2002).

Por ser um modelo relativamente novo na Psicologia, a avaliação assistida ainda tem problemas, segundo Haywood e Tzuriel (2002) e Sternberg e Grigorenko (2002): a) problemas psicométricos, como a questão da fidedignidade, que é alta apenas quando se utilizada no formato estático (sem a mediação); b) é necessário ainda saber como estabelecer a validade da avaliação assistida; c) esta modalidade de avaliação não é ensinada nos cursos de graduação, mas somente em workshops; d) consome muito tempo; e) exige julgamento subjetivo para determinar: quais funções cognitivas estão deficitárias e requerem mediação, que tipo de mediação dispensar, quando a mediação não é mais necessária e como interpretar diferenças no desempenho pré e pós-mediação. Diante do grau de subjetividade desse tipo de avaliação, é importante fazer o cálculo de concordância entre examinadores, segundo autores como Haywood e Tzuriel (2002) e Tzuriel e Samuels (2000). 
Compreender a proposta da avaliação assistida é mais fácil do que aplicá-la, sendo ainda pouco utilizada pelos psicólogos educacionais, apesar da influência das visões construtivistas e interacionistas da aprendizagem e do comportamento em vários países. Os fatores da pouca adesão decorrem dos problemas indicados anteriormente e se associam à permanência de velhas concepções sobre estrutura e natureza da inteligência humana por parte de psicólogos e educadores, que ainda consideram que o potencial de aprendizagem é fixo, que conhecimento é sinônimo de fatos que devem ser aprendidos e examinados, e que negligenciam os aspectos afetivos da aprendizagem. Há também as próprias condições práticas para o uso da avaliação assistida, como a falta de tempo dos psicólogos escolares para atendimento individualizados e o pequeno suporte profissional destes ao pessoal da escola, que não espera um relatório com este tipo de dado, não sabendo como interpretar os dados ou as recomendações (BURNS, 1996; DEUTSCH; REYNOLDS, 2000; ELLIOTT; LAUCHLAN; STRINGER, 1996; MEYERS, 1989; STRINGER; ELLIOT; LAUCHLAN, 1997; TZURIEL, 2001; TZURIEL; HAYWOOD, 1992).

Stringer, Elliot e Lauchlan (1997), em particular, consideram que o aspecto central da prática dos psicólogos educacionais deve enfatizar os processos cognitivos da aprendizagem, baseando-se na crença de que a aprendizagem é um processo social. Dessa forma, acreditam que a "[...] avaliação assistida representa a zona de desenvolvimento proximal para os psicólogos educacionais" (p. 239).

Uma posição conciliadora, aqui defendida, considera a avaliação assistida como uma abordagem complementar à avaliação padronizada, normativa, de modo a retratar um quadro holístico e acurado do funcionamento cognitivo, especialmente para pessoas com necessidades educativas especiais, uma vez que cada abordagem responde a questões diferentes, mas complementares (CAMPIONE, 1989; HAYWOOD; TZURIEL, 2002; LIDZ, 1991; LINHARES, 1995; TZURIEL, 2001).

Diante dos dados mostrando a adequação da avaliação assistida para vários tipos de populações, pode-se considerar que o investimento na capacitação de profissionais de Psicologia nesta área contribuiria para mudanças no quadro da inclusão escolar de crianças com necessidades educativas especiais, na medida em que estas teriam suas características melhor avaliadas. Contribuiria, assim, na identificação de necessidades instrucionais mais adequadas e oferece informações específicas sobre suas possibilidades de mudança, quando expostas à situação de treino, auxiliando o processo de intervenção. Um procedimento avaliativo mais prescritivo, por sua vez, auxiliaria o trabalho do professor nas adaptações curriculares necessárias, podendo, inclusive, alterar suas expectativas, em geral baixas, sobre a capacidade de aprendizagem dessas crianças. É possível, assim, obter reflexos na interação social das pessoas que convivem com a criança, afetando, por conseguinte, a maneira da criança interagir e atuar no contexto escolar inclusivo. 


\section{REFERÊNCIAS}

ALMEIDA, L.S. Considerações em torno da medida da inteligência. In: PASQUALI, L. (Org.). Teoria e métodos de medida em Ciências do Comportamento. Brasília: Laboratório de Pesquisa em Avaliação e Medida/Instituto de Psicologia/UnB: INEP, 1996. p. 196-225.

AMARAL, L.A. Conhecendo a deficiência em companhia de Hércules. São Paulo: Robel Editorial, 1995.

AMIRALLIAN, M.L.T.M. O psicólogo e a pessoa com deficiência. In: MASINI, E.A.F.S. et al. (Orgs.). Deficiência: alternativas de intervenção. São Paulo: Casa do Psicólogo, 1997. p. 31-52.

AUSUBEL, D.P., HANESIAN, H.; NOVACK, J.D. Psicologia educacional. Rio de Janeiro: Interamericano, 1978.

BATISTA, C.G., HORINO, L.E., NUNES, S.S. Avaliação assistida de habilidades cognitivas em crianças com deficiência visual e com dificuldades de aprendizagem. Psicologia: Reflexão e Crítica, Porto Alegre, v. 17, n. 3, p. 381-393, 2004.

BATISTA, M.W.; ENUMO, S.R.F. Inclusão escolar e Deficiência Mental: análise da interação social entre companheiros. Estudos de Psicologia, Natal, v. 9, n.1, p. 101-111, 2004.

BENJAMIN, L; LOMOFSKY, L. The effects of observation of dynamic and static asessement on teachers' perception of learners with low academic results. Journal of Cognitive Education and Psychology, v. 2, n. 2, p. 102-123, 2002.

BORDIN, M.B.M. Desenvolvimento psicológico na fase escolar de criança ex-prematura, diferenciadas quanto à gravidade do risco neonatal. 2005. Tese (Doutorado em Psicologia) - Programa de Pós-Graduação em Psicologia, Universidade de São Paulo, Ribeirão Preto, SP.

BORDIN, M.B.M., LINHARES, M.B.M.; JORGE, S.M. Aspectos cognitivos e comportamentais na média meninice de crianças nascidas pré-termo e com muito baixo peso. Psicologia: Teoria e Pesquisa, Brasília, v. 17, n.1, p. 49-57, 2001.

BUDOFF, M. The validity of Learning Potential Assessment. In: LIDZ, C.S. (Ed.). Dynamic assessment: an interactional approach to evaluating learning potential. New York: The Guilford Press, 1987. p. 52-81

BURNS, M.S. Dynamic assessment: easier said than done. In: LUTHER, M.; COLE, E.; GAMLIN, P. (Eds.). Dynamic assessment for instruction: from theory to application. New York: Captus University Publications, 1996. p. 182-188.

CAMPIONE, J.C. Assisted assessment: a taxonomy of approaches and an outline of strengths and weaknesses. Journal of Learning Disabilities, v. 22. n. 3, p. 151-165, 1989.

CAMPIONE, J.; BROWN, A.L. Linking dynamic assessment with school achievement. In: LIDZ, C.S. (Ed.). Dynamic assessment: an interactional approach to evaluating learning potential. New York: The Guilford Press, 1987. p. 82-115.

CARDOSO, M.C.F. Integração educacional e comunitária. Revista Brasileira de Educação Especial, Piracicaba, v.1, n. 1, p. 89-99, 1992.

CARLSON, J.S., WIEDL, K. The dynamic assessment of intelligence. In: HAYWOOD, H.C.; TZURIEL, D. (Eds.), Interactive assessment. New York: Springer-Verlag, 1992. p. 167-186.

CARVALHO, R.E. Panorama internacional da integração: enfoque nacional. Revista Integração, Brasília, v.5, n. 11, p. 9-13, 1994. 
CARNEIRO, E.G.P.; FERREIRA, I.C.N. Avaliação da inteligência nas pesquisas brasileiras segundo diferentes modelos: a situação atual. Arquivos Brasileiros de Psicologia, Rio de Janeiro, v. 44, n. 3 e 4, p. 157-194, 1992.

COLL, C.; ONRUBIA, J. Inteligência, aptidões para Aprendizagem e rendimento Escolar. In: COOL, C.; PALACIOS, J.; MARCHESI, A. (Orgs.). Desenvolvimento Psicológico e Educação: Psicologia da Educação. Tradução de A. M. Alves. Porto Alegre: Artes Médicas, 1996. v. 2, p. 141-153.

CUNHA, A.C.B. Avaliação cognitiva de crianças com baixa visão leve por procedimentos tradicional e assistido e suas relações com comportamentos e mediação materna. 2004. Tese (Doutorado em Psicologia) - Programa de Pós-Graduação em Psicologia, Universidade Federal do Espírito Santo, Vitória, ES.

CUNHA, A.C.B.; ENUMO, S.R.F. Desenvolvimento da criança com Deficiência Visual (DV) e interacção mãe-criança: algumas considerações. Psicologia Saúde Doenças, Lisboa, v. 4, n. 1, p. 33-46, 2003.

CUNHA, A.C.B.; ENUMO, S.R.F.; PEDROZA, C.P. Será que o meu filho tem potencial de aprendizagem: breve análise da relação cognitiva dinâmica da criança com deficiência visual (D.V.) e interacção mãe-criança. Integrar, Lisboa, v. 21-22, no. especial, p. 47-52, 2004.

DELCLOS, V.R.; BURNS, M.S.; KULEWICZ, S.J. Effects of dynamic assessment on teachers'expectations of handicapped children. American Educational Research Journal, v. 24, n. 3, p. 325-336, 1987.

DELCLOS, V.R.; BURNS, M.S.; VYE, N.J. A comparasion of teachear's response to dynamic and traditional assessment reports. Journal of Psychoeducational Assessment, v. 11, p. 46-65, 1993.

DEUTSCH, R.; REYNOLDS, Y. The use of dynamic assessment by educational psychologists in the UK. Educational Psychology in Practice, v. 16, n. 2, p. 311-331, 2000.

DIAS, T.L. Criatividade em crianças com dificuldade de aprendizagem: Avaliação e intervenção através de procedimentos tradicional e assistido. 2004. Tese (Doutorado em Psicologia), Universidade Federal do Espírito Santo, Vitória, ES.

DIAS, T.L.; ENUMO, S.R.F. Avaliação dinâmica: Uma proposta alternativa e complementar de avaliação cognitiva em crianças com indicação de dificuldade de aprendizagem. In: REUNIÃO ANUAL DA ASSOCIAÇÃ̃O NACIONAL DE PESQUISA E PÓS-GRADUAÇÃO EM EDUCAÇÃO, 27., 2004, Caxambu. Anais ... Caxambú, MG: ANPED, 2004. 1 CD-ROM, 17 p.

DIAS, T.L., ENUMO, S.R.F., PEREIRA, M.J.S.B., RABBI, J.S., RIBEIRO, M.P.L. Desempenho acadêmico e cognitivo de alunos com indicação de dificuldade de aprendizagem: uma análise após programa de intervenção. In: REUNIÃO ANUAL DE PSICOLOGIA, 33., 2003, Belo Horizonte. Resumos de Comunicações Científicas. Belo Horizonte, MG: Sociedade Brasileira de Psicologia, 2003. p. 221-222.

DIAS, T.L.; PAULA, K.M.P.; FERRÃO, E.S.; ENUMO, S.R.F. Avaliação assistida: contribuições a programas de intervenção para crianças com necessidades educativas especia2is. In: ALCHIERI, J.C.; CRUZ, J.C. (Orgs.). Avaliação psicológica no contexto iberoamericano. Buenos Aires: Lúmen, no prelo. 
ELLIOT, J.; LAUCHLAN, F.; STRINGER, P. Dynamic assessment and its potential for educational psychologists- Part 1- theory and practice. Educational Psychology in Practice, v. 12, n. 3, p. 152-160, 1996.

ENUMO, S.R.F.; BATISTA, C.G. Evaluation of cognitive abilities of visually impaired children. In: STUEN, C.; ARDITI, A.; HOROWITZ, A.; LANG, M.A.; ROSENTHAL, B.; SEIDMAN, K.R. (Eds.). Vision Rehabilitation: assessment, intervention and outcomes. New York: Swets \& Zeitlinger, 2000. p. 379-381.

ENUMO, S.R.F.; BATISTA, C.G.; FERRÃO, E.S. Uma proposta de avaliação de aspectos do desenvolvimento cognitivo e acadêmico de crianças com Deficiência Visual. In: QUEIROZ, S.S.; ENUMO, S.R.F.; ORTEGA, A.C. (Orgs.). Desenvolvimento humano e aprendizagem: temas contemporâneos. Vitória: Programa de Pós-Graduação em Psicologia/CAPES; Linhares: Unilinhares, 2005, p. 45-78.

ENUMO, S.R.F.; BATISTA, C.G.; FERRÃO, E.S.; FERREIRA, B.S. Habilidades cognitivas em crianças com deficiência visual: análise de uma proposta de avaliação. In: REUNIÃO DA SOCIEDADE BRASILEIRA PARA O PROGRESSO DA CIÊNCIA, 52., 2000, Brasília. Anais .... Brasília: SBPC, 2000. 1 CD-ROM.

ENUMO, S.R.F.; CUNHA, A.C.B. Intervenções comportamentais e cognitivas em pessoas com deficiências. In: RANGÉ, B. (Org.). Psicoterapias cognitivo-comportamentais: um diálogo com a Psiquiatria. Porto Alegre: ArtMed, 2001. cap. 30, p. 499-511.

ENUMO, S.R.F.; CUNHA, A.C.B.; PAULA, K.M.P.; DIAS, T.L. Comportamentos do mediador e da criança com deficiência visual na avaliação assistida de habilidades cognitivas. Temas em Psicologia, Ribeirão Preto, v. 10, n. 1, p. 71-84, 2002.

ENUMO, S.R.F.; LINHARES. M.B.M.; ESCOLANO, A.C.M. Avaliação cognitiva assistida: fundamentos teóricos-conceituais, procedimentos de avaliação e aplicabilidade. In: REUNIÃO ANUAL DE PSICOLOGIA DA SOCIEDADE BRASILEIRA DE PSICOLOGIA, 34., 2004, Ribeirão Preto. Resumos de Comunicação Científica. Ribeirão Preto, SP: Sociedade Brasileira de Psicologia, 2004. 1 CD-ROM.

ESCOLANO, A.C.M. Avaliação cognitiva assistida em situação de resolução de problema na predição do desempenho escolar de crianças de primeira série do Primeiro Grau. 2000. Dissertação (Mestrado em Psicologia) - Programa de Pós-Graduação em Psicologia, Universidade de São Paulo, Ribeirão Preto, SP.

Fatores de risco e mecanismos de proteção na trajetória de desenvolvimento de escolares de primeira à quarta série do ensino fundamental. 2004. Tese (Doutorado em Psicologia) - Programa de Pós-Graduação em Psicologia, Universidade de São Paulo. Ribeirão Preto, SP.

ESCOLANO, A.C.M.; LINHARES, M.B.M. Assistida cognitiva assistida em situação de resolução de problema na predição do desempenho escolar de crianças de primeira série do primeiro grau. In: ALVES, Z.M.M.B.; JAPUR, M.; SANTOS, M.A. (Orgs.). Livro de artigos do III Seminários de Pesquisa - Tomo II (pp. 55-63). Ribeirão Preto: Programa de Pós-graduação em Psicologia, Universidade de São Paulo, 2000. p. 55-63.

FERRÃO, E.S.; ENUMO, S.R.F.; LINHARES, M.B.M.; RODRIGUES, C.G.; MAIA, C.C.; SOUSA, G.P.; ORLANDI, E.; SANTOS, R.F.; PAULA, K.M.P. Avaliação de aspectos afetivomotivacionais em provas cognitivas assistidas: construindo categorias comportamentais para crianças com necessidades especiais. In: REUNIÃO ANUAL DE PSICOLOGIA, 35., 
2005, Curitiba. Resumos de Comunicações Científicas. Curitiba, PR: Sociedade Brasileira de Psicologia, 2005. 1 CD-ROM.

FERRIOLLI, S.H.T. Indicadores de potencial cognitivo de crianças com queixa de dificuldade de aprendizagem obtidos através da avaliação assistida. 2000. Dissertação (Mestrado em Psicologia) - Programa de Pós-Graduação em Psicologia, Universidade de São Paulo, Ribeirão Preto, SP.

FERRIOLLI, S.H.T.; LINHARES, M.B.M.; LOUREIRO, S.R.; MARTURANO, E.M. Indicadores de potencial de aprendizagem obtidos através da avaliação assistida. Psicologia: Reflexão e Crítica, Porto Alegre, v. 14, n. 1, p. 35-43, 2001.

FEUERSTEIN, R. The dynamic assessment of retarded performers. Baltimore: University Park Press, 1979.

FONSECA, V. Cognição e aprendizagem: abordagem neuropsicológica e psicopedagógica. Lisboa: Âncora, 2001.

FONSECA, V.; CUNHA, A.C.B. Teoria da experiência de aprendizagem mediatizada e interação familiar: prevenção das perturbações do desenvolvimento e aprendizagem. Lisboa, Portugal: Editora da Faculdade de Motricidade Humana, 2003.

FONSECA, V.; CUNHA, A.C.B.; ENUMO, S.R.F. O desenvolvimento cognitivo da criança com deficiência visual e suas perspectivas de avaliação: da abordagem padronizada à avaliação dinâmica. Revista de Educação Especial e Reabilitação. Lisboa, Portugal, v. 9, n. 1-2, p. 75-91, 2002.

GERA, A. Estratégias de pergunta de busca de informações na resolução e problemas em situação de avaliação assistida de crianças com queixa de dificuldade de aprendizagem. 2001. Dissertação (Mestrado em Psicologia) - Programa de Pós-Graduação em Psicologia, Universidade de São Paulo, Ribeirão Preto, SP.

GLAT, R. A integração social dos portadores de deficiências: uma reflexão. Rio de Janeiro: Sette Letras, 1998.

GOFFMAN, E. Estigma: notas sobre a manipulação da identidade deteriorada $4^{\mathrm{a}}$ ed.. Rio de Janeiro: Zahar, 1982.

GUTHKE, J.; WINGENFEL, S. The Learning Test concept: origins, state of the art, and trends. In: HAYWOOD, H.C.; TZURIEL, D. (Eds.), Interative assessment (pp. 64-93). New York: Springer-Verlag, 1992. p. 64-93

HAYWOOD, H.C.; MILLER, M.B. Dynamic assessment of adults with traumatic brain injuries. Journal of Cognitive Education and Psychology, v. 3, p. 137-163, 2003. Disponível em: <http://www.iacep.coged.org>

HAYWOOD, H.C.; TZURIEL, D. (Eds.). Interative assessment. New York: Springer-Verlag, 1992.

HAYWOOD, H.C.; TZURIEL, D. Applications and challenges in dynamic assessment. Peabody Journal of Education, v. 77, n. 2, p. 40-63, 2002.

HAYWOOD, C.H.; TZURIEL, D.; VAUGHT, S. Psychoeducational assessment from a transactional perspective. In: HAYWOOD, C.H.; TZURIEL, D. (Eds.). Interative assessment (p. 33-63). New York: Springer-Verlag, 1992. p. 33-63. 
HUTZ, C.; BANDEIRA, D. Tendências contemporâneas no uso de testes: uma análise da literatura brasileira e internacional. Psicologia: Reflexão e Crítica, Porto Alegre, v. 6, n. 1/2, p. 85-101, 1993.

KARAGIANNIS, A.; STAINBACK, S.; STAINBACK, W. Visão histórica da inclusão. In: STAINBACK, S.; STAINBACK, W. (Orgs.), Inclusão: um guia para educadores Tradução de M. F. Lopes. Porto Alegre: ArtMed, 1999a. p. 35-47.

KARAGIANNIS, A.; STAINBACK, S.; STAINBACK, W. Fundamentos do ensino inclusivo. In: STAINBACK, S.; STAINBACK, W. (Orgs.), Inclusão: um guia para educadores Tradução de M. F. Lopes. Porto Alegre: ArtMed, 1999b. p. 21-34.

KLEIN, V.C. Interação entre mães e crianças pré-escolares nascidas pré-termo com muito baixo peso em situações lúdicas e de ensino. 2005. Dissertação (Mestrado em Saúde Mental). Faculdade de Medicina de Ribeirão Preto-USP. Ribeirão Preto, SP.

LEITE, L.; MOTTA, A.B.; ORLANDI, E.; ENUMO, S.R.F.; RODRIGUES, M.M.P. Contar histórias: avaliação da narrativa por procedimento assistido, em pré-escolares. In: SOCIEDADE BRASILEIRA PARA O PROGRESSO DA CIÊNCIA, 56., 2004, Cuiabá. Anais.... Cuiabá, MT: SBPC, 2004. 1 CD-ROM.

LIDZ, C.S. (Ed.). Dynamic assessment: an interactional approach to evaluating learning potential. New York: The Guilford Press, 1987. . Practioner's guide to dynamic assessment. New York: The Guilford Press, 1991.

. Dynamic assessment approaches. In: FLANAGAN, D.P.; GENSHAFT, J.L.; HARRISON, P.L. (Eds.). Contemporary intellectual assessment: theories, tests, and issues. New York: The Guilford Press, 1996. p. 281-296.

LINHARES, M.B.M. Avaliação Assistida: fundamentos, definição, características e implicações para a avaliação psicológica. Psicologia: Teoria e Pesquisa, Brasília, v. 11, n. 1, p. 23-31, 1995.

. Avaliação assistida em crianças com queixa de dificuldade de aprendizagem. Temas em Psicologia, Ribeirão Preto, v. 4, n. 1, p.17-32, 1996.

. Avaliação assistida de crianças com queixa de dificuldade de aprendizagem: Indicadores de eficiência e transferência de aprendizagem em situação de resolução de problema. In: ZUARDI, A.W.; MARTURANO, E.M.; M.A.C. FIGUEIREDO, M.A.C.; LOUREIRO, S.R. (Orgs.). Estudos em Saúde Mental. Ribeirão Preto: Curso de Pós-Graduação em Saúde Mental- Faculdade de Medicina de Ribeirão Preto, Universidade de São Paulo, 1998. p. 121-147.

LINHARES, M.B.M.; BORDIN, M.B.M.; CARVALHO, A.E.V. Aspectos do desenvolvimento psicológico da criança ex-prematura na fase escolar. In: MARTURANO, E.M.; LINHARES, M.B.M.; LOUREIRO, S.R. (Orgs.). Vulnerabilidade e proteção: indicadores na trajetória de desenvolvimento do escolar. São Paulo: Casa do Psicólogo, 2004. p. 75-106.

LINHARES, M.B.M.; ESCOLANO, A.C.M.; ENUMO, S.R.F. (Orgs.). Avaliação cognitiva assistida: fundamentos teórico-conceituais e contribuições. São Paulo: Casa do Psicólogo, no prelo. 
LINHARES, M.B.M.; ENUMO, S.R.F. Avaliação assistida de crianças no Brasil. In: ALCHIERI, J.C.; CRUZ, J.C. (Orgs.). Avaliação psicológica no contexto iberoamericano. Buenos Aires: Lúmen, no prelo.

LINHARES, M.B.M.; MARTINS, I.M.B.; KLEIN, V.C. Mediação materna como processo de promoção e proteção do desenvolvimento da criança nascida prematura. In: MARTURANO, E.M.; LINHARES, M.B.M.; LOUREIRO, S.R. (Orgs.). Vulnerabilidade e proteção: indicadores na trajetória de desenvolvimento do escolar. São Paulo: Casa do Psicólogo, 2004. p. 39-74.

LINHARES, M.B.M.; SANTA MARIA, M.R.; ESCOLANO, A.A.C.M.; GERA, A.A. Avaliação assistida: uma abordagem promissora na avaliação cognitiva de crianças. Temas em Psicologia, Ribeirão Preto, v. 6, n. 3, p. 231-254, 1998.

MACHADO, A.M.; SOUZA, M.P.R.; SAYÃO, Y. As classes especiais e uma proposta de avaliação psicológica. In: CONSELHO REGIONAL DE PSICOLOGIA (Org.). Educação Especial em debate. São Paulo: Casa do Psicólogo, Conselho Regional de Psicologia, 1997. p. 69-116.

MARCHESI, A.; ECHEITA, G., MARTÍN, E. A avaliação da integração. In: COLL, C.; MARCHESI, A.; PALACIOS, J. (Orgs.). Desenvolvimento psicológico e educação: necessidades educativas especiais e aprendizagem escolar. Tradução de M.A.G. Domingues. Porto Alegre: Artes Médicas, 1995. v. 3, p. 336-357.

MARCHESI, A.; MARTÍN, E. Da terminologia do distúrbio às necessidades educativas especiais. In: COLL, C.; MARCHESI, A.; PALACIOS, J. (Orgs.). Desenvolvimento psicológico e educação: necessidades educativas especiais e aprendizagem escolar. Tradução de M.A.G. Domingues. Porto Alegre: Artes Médicas, 1995. v. 3, p. 7-23.

MARTÍN, E.; MARCHESI, A. Desenvolvimento metacognitivo e problemas de aprendizagem. In: COLL, C.; MARCHESI, A.; PALACIOS, J. (Orgs.). Desenvolvimento psicológico e educação: necessidades educativas especiais e aprendizagem escolar. Tradução de M.A.G. Domingues. Porto Alegre: Artes Médicas, 1995. v. 3, p. 24-35.

MENDES, E.G.; FERREIRA, J.R.; NUNES, L.R.O.P. Integração/inclusão: o que revelam as teses e dissertações em Educação e Psicologia. In: NUNES SOBRINHO, F.P. (Org.). Inclusão educacional: pesquisa e interfaces. Rio de Janeiro: Livre Expressão, 2003. p. 98-149.

MEYERS, J. The training of dynamic assessment. In: LIDZ. C.S. (Ed.). Dynamic assessment: an interactional approach to evaluating learning potential. New York: The Guilford Press, 1989. p. 403-425.

MILLER, L.; GILLAM, R.B.; PEÑA, E.D. Dynamic assessment and intervention: improving children's narrative abilities. Austin, Texas: Proed, 2001.

MOTTA, A.B.; ENUMO, S.R.F.; LEITE, L.; ORLANDI, E., MAIA, C.C.; DIAS, T.L.; RODRIGUES, M.M.P. Telling stories: a proposal of dynamic assessment of the narrative in children with cancer. In: CONFERENCE OF THE INTERNATIONAL ASSOCIATION FOR COGNITIVE EDUCATION AND PSYCHOLOGY, 2005, 10., Durham, UK, Proceeding...Durham, UK: IACEP, p. 88.

MOFESE, V.J.; ACHESON, S. Infant and preschool mental and verbal abilities: how are infant scores related to preschool score? The International Society for the Study of Behavioral Development, v. 20, n. 4, p. 595-607, 1997. 
NUNES, L.R.O.P.; GLAT, R.; FERREIRA, J.R.; MENDES, E.G. Pesquisa em Educação Especial na Pós-Graduação. Rio de Janeiro. Sette Letras, 1998.

ORLANDI, E.; FERRÃO, E.S.; LEITE, L.; ENUMO, S.R.F. Uma proposta de avaliação de fatores não-intelectuais do desempenho de crianças em provas assistidas de habilidades cognitivas. In: JORNADA DE INICIAÇÃO CIENTÍFICA DA UNIVERSIDADE FEDERAL DO ESPÍRITO SANTO, 14., 2004, Vitória. Cadernos de Resumos. Vitória, ES: UFES, 2004. p. 61.

PAOUR, J. Induction of logic structures in the mentally retarded: an assessment and intervention instrument. In: HAYWOOD, H.C.; TZURIEL, D. (Eds.), Interative assessment. New York: Springer-Verlag, 1992. p. 119-166.

PAULA, K.M.P. Avaliação assistida: análise de indicadores cognitivos, comportamentais e afetivomotivacionais em crianças na situação de intervenção com sistema de comunicação alternativa. 2004. Tese (Doutorado em Psicologia) - Programa de Pós-Graduação em Psicologia, Universidade Federal do Espírito Santo, Vitória, ES.

PAULA, K.M.P.; ENUMO, S.R.F.; FERRÃO, E.S.; GOTTARDI, A.P.; TURINI, F.A. Inclusão escolar: recursos da comunicação alternativa e ampliada para expandir habilidades de linguagem e cognição em crianças com desordens de comunicação. In: REUNIÃO ANUAL DA SOCIEDADE BRASILEIRA DE PSICOLOGIA, 33., 2003, Belo Horizonte. Resumos de Comunicação Científica. Belo Horizonte: SBP, 2003. p. 211.

PAULA, K.M.P.; ENUMO, S.R.F.; MAIA, C. Acquisition and use of AAC in brazilian school context through the dynamic assessment. In: BIENNIAL CONVENTION OF INTERNATIONAL SOCIETY FOR AUGMENTATIVE AND ALTERNATIVE COMMUNICATION, 11., 2004, Natal. Proceeding... Natal, RN: ISAAC, 2004. 1 CD-ROM.

PEARCE, J. Identificação dos distúrbios psiquiátricos na criança. In: GARRALDA, M.E. (Org.), Tratando a criança com problemas psiquiátricos: manual para médicos, psicólogos, pais e educadores. Tradução de H.T. Buckup. São Paulo: Livraria Santos Editora, Maltese, 1995. p. 3-21.

PEREIRA, M.J.S.B.; DIAS, T.L.; ENUMO, S.R.F. Análise da mediação do aplicador durante a avaliação cognitiva dinâmica de alunos com indicação de dificuldade de aprendizagem. In: Seminário Capixaba de Educação Inclusiva, 7., 2003, Vitória. Anais... Vitória, ES: UFES, 2003. 1 CD-ROM.

RAND, Y.; KANIEL, S. Group administration of LPAD. In: LIDZ, C.S. (Ed.). Dynamic assessment: an interactional approach to evaluating learning potential. London: Guilford Press, 1987. p. 196-214.

SAINT-LAURENT, L. A educação de alunos com necessidades especiais. In: MANTOAN, M.T.E. (Org.). A integração de pessoas com deficiência: uma contribuição para uma reflexão sobre o tema. São Paulo: Memnon, 1997. p. 67-76.

SANTA MARIA, M.R.; LINHARES, M.B.M. Avaliação cognitiva assistida de crianças com indicações de dificuldades de aprendizagem escolar e deficiência mental leve. Psicologia: Reflexão e Crítica, Porto Alegre, v. 12, n. 2, p.395-417, 1999.

SASSAKI, R.K. Inclusão: Construindo uma sociedade para todos. Rio de Janeiro: WVA, 1997.

STAINBACK, S.; STAINBACK, W. Observações finais: preocupações com a inclusão. In: (Orgs.). Inclusão: um guia para educadores. Tradução de M.F. Lopes. Porto Alegre: ArtMed., 1999. p. 432-435. 
STERNBERG, R.J.; GRIGORENKO, E.L. Dynamic testing: the nature and measurement of learning potential. New York: Cambridge University Press, 2002.

STRINGER, P.; ELLIOTT, J.; LAUCHLAN, F. Dynamic assessment and its potencial for educational psychologists - Part 2 - The zone of next development? Educational Psychology in Practice, v. 12, n. 4, p. 234-239, 1997.

TZURIEL, D. Cognitive modifiability, mediated learning experience and a affectivemotivational processes: a transactional approach. In: FEUERSTEIN, R.; P. S. KLEIN, P.S.; TANNENBAUM, A. (Eds.). Mediated learning experience (pp. 95-120). London: Freund, 1991. p. 95-120.

TZURIEL, D. Dynamic assessment of young children. New York: Kluwer Academic/ Plenum Publishers, 2001.

TZURIEL, D.; FEUERSTEIN, R. Dynamic group assessment for prescriptive teaching: differential effects of treatments. In: HAYWOOD, C.H.; TZURIEL, D. (Eds.). Interative assessment. New York: Springer-Verlag, 1992. p. 187-206.

TZURIEL, D.; HAYWOOD, H.A. The development of interactive-dynamic approaches to assessment of learning potential. In: HAYWOOD, C.H.; TZURIEL, D. (Eds.). Interative assessment. New York: Springer-Verlag, 1992, P. 3-37.

TZURIEL, D.; SAMUELS, M.T. Dynamic assessment of learning potential: inter-rater reliability of deficient cognitive functions, types of mediation, and non-intellective factors. Journal of Cognitive Education and Psychology, v. 1, p. 2-23, 2000. Disponível em: <http:// www.iacep.coged.org>.

TZURIEL, D.; SHAMIR, A. The effects of mediation in computer assisted dynamic assessment. Journal of Computer Assisted Learning, v. 18, p. 21-32, 2002.

UTLEY, C.A.; HAYWOOD, H.C.; MASTERS, J. C. Policy implications of psychological assessment of minority children. In: HAYWOOD, H.C.; TZURIEL, D. (Eds.). Interative assessment. New York: Springer-Verlag, 1992. p. 445-469.

$\overline{\text { Recebido em } 07 / 07 / 2005}$

Reformulado: 10/10/2005

Aceito em 22/11/2005 